Communications in Physics, Vol.21, No. 3 (2011), pp. 219-224

\title{
ON THE INFORMATION PROBLEM OF DNA IN THE DENATURATION PROCESS
}

\author{
VI THI HA MY, VU THUY HUONG, CHU THUY ANH, TO THI THAO, \\ NGO VAN THANH, AND NGUYEN AI VIET \\ Institute of Physics, VAST
}

\begin{abstract}
The information problem of DNA in the denaturation process was studied by the relationship between entropy and information. Using the previous PBs (Peyrard and Bishop) numerical results obtained from their microscopic arharmonic model of DNA, we propose a new effective simple model to calculate DNA information. A shape change of information at the denaturation phase transition point was found and discussed.
\end{abstract}

\section{INTRODUCTION}

The nature of evolution of life is an interesting problem that is studied by scientists for a long time. In the research process, one answer was found out that the general struggle for existence of animate beings is not a struggle for raw materials, for organisms, air, water and soil, all abundantly available nor for energy which exists in plenty in any body in the form of heat, but a struggle for entropy (Boltzmann, 1886) [8]. For this reason, living systems, from cells to the biosphere have spontaneously increased in complexity in order to increase their total entropy production. This issue has been proven and developed in many articles. In 1972, Prigogine and co-workers showed that non-equilibrium structuring of matter in space and time, from molecules to hurricanes to living systems, is contingent on entropy production[9]. Paltridge continued with the article Climate and thermodynamic systems of maximum dissipation in 1979 to demonstrate that Nature tends to find new routs to increasing entropy production, whether abiotic, biotic, or mixed abiotic-biotic, and this has been referred to as the maximum entropy production principle [10]. After that, K. Michaelian used this theory to interpret the reprodution of RNA and DNA, the process building life [11]. Thus, it can be said that entropy production is the force of life evolution. However, does the life simply operate that way or is there any rule to control it? Entropy is related to information, is there thus any change in information during entropy changing?

The link between entropy and information has been made by Shannon and Weaver (1949). If the probabilities in question are the thermodynamic probabilities pi; then the (reduced) Gibbs entropy can be seen as the amount of Shannon information needed to give the macroscopic description of the system. Hence, when the entropy of a system changes, there must be a change in information. For this reason, in this paper we will study the information of a specific system, DNA, in its denaturation process. 
The separation of double helix structure of DNA is an important starting point in the informatics replication process of DNA that needs to reproduce the living matter. Accordingly, the problem of DNA denaturation has investigated extensively step by step in various aspects. Englander et al.[6] first suggested a theory of soliton excitations in oder to explain the open states of DNA (1980). Later, in 1989, Peyrard and co-workers proposed a simple lattice model [Peyrard-Bishop (PB) model] for the denaturation of the DNA double helix [3] and determined the temperature dependence of the interstrand seperation and explained some experimental results on DNA. Furthermore, this studying was expanded with more complicated models and the more thermodynamical properties was found out [4]. However, there has been no research studying the information of DNA. In this paper, we use these DNA models to study another aspect of the denaturation process: the information problem.

\section{BOGOLIBOV DIAGONALIZATION METHOD}

Considering a physical system in which has two interactive energy levels $E_{1}$ and $E_{2}$, the interactive Hamiltonian of the system is:

$$
H=E_{1} a^{+} a+E_{2} b^{+} b+g\left(a^{+} b+b^{+} a\right)
$$

where $\mathrm{g}$ is interaction coefficient.

Using the Bogolibov method, we can transform $\mathrm{H}$ into diagonalized form.

$$
H=\varepsilon_{1} \gamma_{1}^{+} \gamma_{1}+\varepsilon_{2} \gamma_{2}^{+} \gamma_{2}
$$

$\varepsilon_{1}, \varepsilon_{2}$ is roots of the equation:

$$
\left|\begin{array}{cc}
E_{1}-\varepsilon & g \\
g & E_{2}-\varepsilon
\end{array}\right|=0
$$

It yields:

$$
\begin{aligned}
& \varepsilon_{1}=\frac{E_{1}+E_{2}-\sqrt{\left(E_{1}-E_{2}\right)^{2}+4 g^{2}}}{2} \\
& \varepsilon_{2}=\frac{E_{1}+E_{2}+\sqrt{\left(E_{1}-E_{2}\right)^{2}+4 g^{2}}}{2}
\end{aligned}
$$

Instead of calculating with $E_{1}, E_{2}$, we can use $\varepsilon_{1}, \varepsilon_{2}$ to work easier.

\section{DNA'S INFORMATION}

In this section, we analyze the DNA's information for PB's model in two cases: the harmonic potential and the anharmonic potential. Firstly, we start with the harmonic case. The Hamiltonian for the model in this case is:

$$
H=\sum_{n}\left[\frac{1}{2} m \dot{y}_{n}+D\left(e^{-\alpha y_{n}}-1\right)^{2}+\frac{1}{2} K\left(y_{n}-y_{n-1}\right)^{2}\right.
$$

where $\mathrm{m}$ is the common mass for the bases; $V=D\left(e^{-\alpha y_{n}}-1\right)^{2}$ is the Morse potential and $\mathrm{K}$ is the coupling constant along each strand. 
Calculating the partition function of a chain containing $\mathrm{N}$ base pairs, we obtain:

$$
Z=Z_{p} Z_{y}
$$

where:

$$
\begin{gathered}
Z_{p}=\left(2 \pi m k_{B} T\right)^{N / 2} \\
Z_{y}=\exp \left(-\mathrm{N} \beta \varepsilon_{0}\right)
\end{gathered}
$$

$\varepsilon_{0}$ here is the lowest eigenvalue of the transfer integral operator [3] and can be obtained exactly from the analytic solution:

$$
\varepsilon_{0}=\frac{1}{2 \beta} \ln \left[\frac{\beta K}{2 \pi}\right]+\frac{\alpha}{\beta}\left[\frac{D}{K}\right]^{1 / 2}-\frac{\alpha^{2}}{4 \beta^{2} K}
$$

Using these result (6), (7), (8), (9) we can compute the free energy $F=-k_{B} T \ln (Z)$ and also the thermodynamic entropy per site $S=-\frac{1}{N} \frac{\partial F}{\partial T}$. It yields:

$$
S=\frac{1}{2} k_{B} \ln (m K)+\frac{\alpha^{2} k_{B}^{2}}{2 K} T-\alpha k_{B}\left(\frac{D}{K}\right)^{1 / 2}
$$

Basing on the theoretical relationship between thermodynamic entropy and information entropy, the DNAs information entropy can be determined by:

$$
I=I_{o}-\frac{1}{2} \ln (m K)+\frac{\alpha^{2} k_{B}}{2 K} T-\alpha\left(\frac{D}{K}\right)^{1 / 2}
$$

in which $I_{O}$ is a constant depending on the system's characteristics.

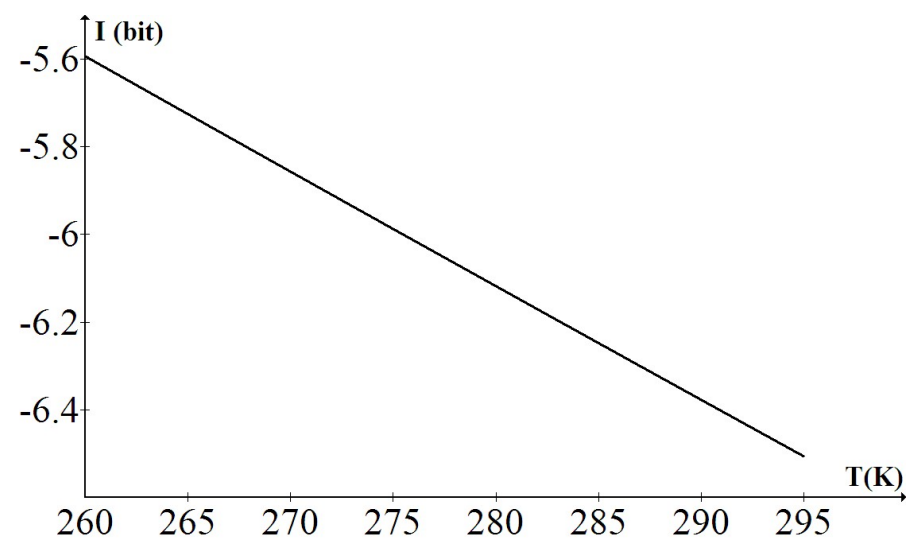

Fig. 1. The evolution of $\mathrm{I}$ versus $\mathrm{T}$ in the harmonic case.

The result is showed in Fig. 1 with the following parameters values: dissociation energy $D=0.03 \mathrm{eV}$, a spatial scale factor of the Morse potential $\alpha=4.5 \AA^{-1}$, a coupling constant $\mathrm{K}=0.06 \mathrm{eV} / \AA^{2}$, and a mass $m=300 \mathrm{amu}$. This figure shows a linear increase of $\mathrm{I}$ vesus $\mathrm{T}$ around the denaturation point. It means that in conjunction with denaturation 
process, there is an information accumulation of the system. The second model we study here is the arharmonic model with the Hamiltonian:

$$
H=\sum_{n}\left[\frac{1}{2} m \dot{y}_{n}+D\left(e^{-\alpha y_{n}}-1\right)^{2}+W\left(y_{n}, y_{n-1}\right)\right]
$$

in which the stacking energy between two neighbouring base pairs is described by the arhamonic potential:

$$
W\left(y_{n}, y_{n-1}\right)=\frac{K}{2}\left(1+\rho e^{-\alpha\left(y_{n}+y_{n-1}\right)}\right)\left(y_{n}-y_{n-1}\right)^{2}
$$

The calculation is similar to the first case. However, in this case $\varepsilon_{0}$ must be found by the numerical solution. It turned out that below $T_{C}$, the transfer integral operator has a discrete eigenvalue $\varepsilon_{0}(\mathrm{~T})$ separated from a continuum, while above $T_{C}$ the discrete eigenvalue has disappeared. Hence, in order to solve the problem, we propose an effective simple model which has two interactive energy levels $E_{1}$ and $E_{2}$, where $E_{1}$ is $\varepsilon_{0}$ and $E_{2}$ is the edge of the continuum. Using the Bogolibov approximation method, we can consider the non-interactive energy levels $\varepsilon_{1}, \varepsilon_{2}$ instead of the levels $E_{1}, E_{2}$.

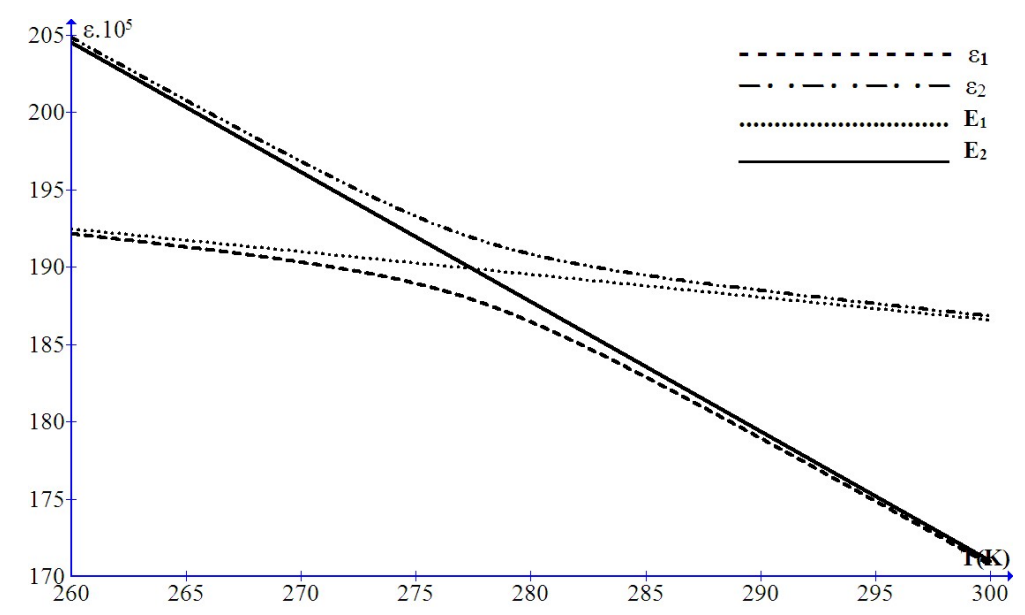

Fig. 2. Two effective eigenvalues $\varepsilon_{1}, \varepsilon_{2}$ calculated by Bogolibov diagonalization method.

The information entropy then is given as follow:

$$
I=\frac{1}{2}+\frac{1}{2} \ln \left(2 \pi m k_{B} T\right)-\frac{1}{k_{B}} \frac{\partial \varepsilon_{1}}{\partial T}
$$

Using the same above parameters values, we point out the evolution of I versus $\mathrm{T}$ around the denaturation temperature $T_{C}$ (Fig. 3 ). The most striking feature is the strong rise in I at denaturation point. This variation also depend on the value of interaction coefficient $g$. Calculating with different values of $g$, we get different rates of variation. When $g=0$, the denaturation process is type-I phase transition, when $g>0$, they are type-II phase transition and in comparison with Fig. 1, the process tend to the harmonic case when $g$ increases. 


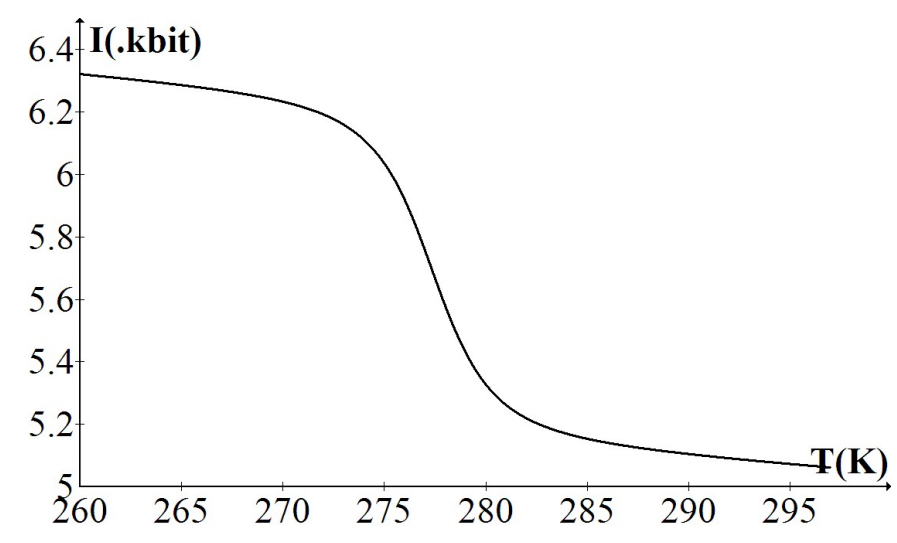

Fig. 3. The evolution of I versus $\mathrm{T}$ in the arharmonic case.

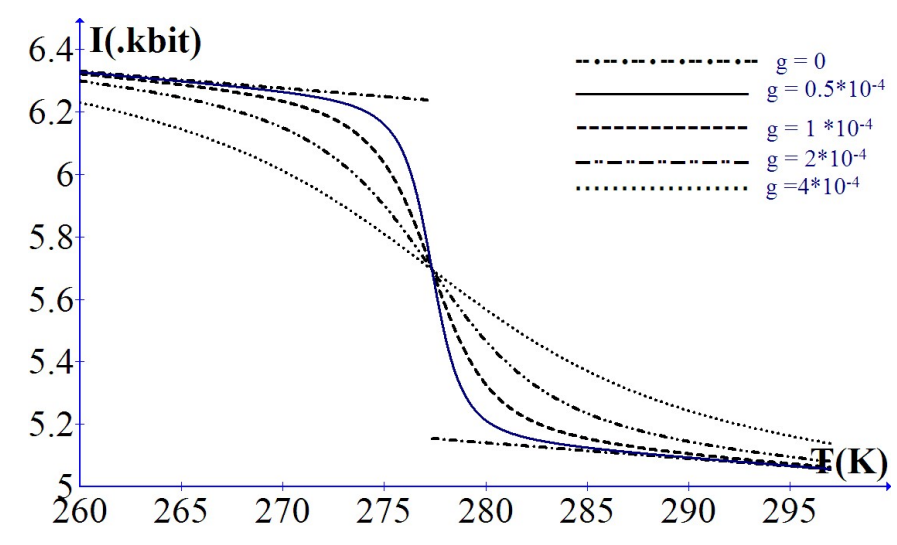

Fig. 4. Variation of DNAs information as a function of the interaction coefficient $g$

\section{CONCLUSION AND DISCUSSION}

The results show that in this model, there is a strong change in DNAs information in the denaturation process. This change depends on the value of the interaction coefficient $g$, when $g=0$, we have type-I phase transition, when $g>0$, they are type-II phase transition. However, the problem is what the changing information is and how about the other DNAs models. It would be interesting to continue this speculation.

\section{REFERENCES}

[1] D. L. Hien, N. T. Nhan, V. Thanh Ngo, and N. A. Viet, Phys. Rev. E76 (2007) 021921.

[2] B. S. Alexandrov, L. T. Wille, K. . Rasmussen, A. R. Bishop, and K. B. Blagoev, Phys. Rev. E74 (2006) 050901(R).

[3] M. Peyrard and A.R. Bishop, Phys. Rev. Lett. 62 (1989) 2755.

[4] T. Dauxois and M. Peyrard, Phys. Rev. E51(1995) 4027.

[5] Nikos Theodorakopoulos, Thierry Dauxois and Michel Peyrard, Phys. Rev. Lett. 85 (2000) 69. 
[6] S. W. Englander, N. R. Kallenback, A. J. Heeger, J. A. Krumhanst and S. Kitwin, Proc. Natl. Acad. Sci. USA. 77 (1980) 7222.

[7] J.A. Krumhansl and J. R. Schrieffer, Phys. Rev. B11 (1975) 3535.

[8] Boltzmann, L. (1886) The Second Law of Thermodynamics

[9] Prigogine, I., Nicolis, G., and Babloyantz A. (1972) Thermodynamics of Evolution (I) Physics Today, 25, 23-28; Thermodynamics of Evolution (II) Physics Today, 25, 38-44.

[10] G.W Paltridge, Nature 279 (1979) 630-631.

[11] K. Michaelian, Journal of Theoretical Biology 237 (2005) 323-335.

Received 14 October 2010. 